\title{
Tendencias de investigación en documentación digital en el ámbito hispano
}

\author{
Francisco Javier García Marco \\ Universidad de Zaragoza (España)
}

\subsection{Resumen}

Se analizan algunos de las tendencias de investigación en documentación digital a partir del análisis y discusión de los estudios publicados en el número dos del volumen 10 de la revista Scire (julio-diciembre 2004), de carácter monográfico. Los artículos constituyen un panorama representativo de las investigaciones sobre documentación digital en el ámbito hispano tanto desde el punto de vista de sus principales campos de aplicación - las Intranets corporativas, las bibliotecas digitales, los portales científicos y periodísticos, las redes de vigilancia tecnológica, los servicios de información administrativa y ciudadana - como en lo que concierne a algunos de los temas transversales más candentes en la actualidad - el desarrollo de modelos de sistemas de información especializados, el software libre, la transición al XML y a la Web semántica, la búsqueda en lenguaje natural, los metadatos, la visibilidad, la usabilidad, los estudios de usuarios y la minería de datos en la Internet - El análisis de los trabajos se concentra en torno a cuatro énfasis: las intranets como instrumento para la gestión del conocimiento, el movimiento del código abierto en el desarrollo de infraestructuras documentales, la revolución digital en la documentación de medios de comunicación, y el papel de la tecnología como elemento dinamizador de proyectos de trabajo en grupo y de animación de redes sociales.

Palabras clave: Tendencias de investigación. Editorial. Revisión. Intranets. Software libre. Medios de comunicación. Administración pública. Redes sociotecnológicas. Méjico. España.

\subsection{Abstract}

Presentation and analysis of some research trends in Information Science research as represented by the articles published in a monographic number of the journal Scire (volume 10, number 1, July-December 2004). The book offers a landscape of the current research on digital documentation in the Hispanic world from the point of view of its main fields of application - Intranets, digital librarScire. $10: 2$ (jul.-dic. 2004) 9-24. 
ies, scientific and media portals, technological monitoring networks, and administrative and citizen information services - and also from the perspective of the crosscutting hot topics of the moment - specialized information system models, metadata, free software, XML and semantic web transition, visibility, usability, user behaviour, Internet data mining, natural language search, etc. - . The analysis and commentary of the articles is focused in four emphases: Intranets as a knowledge management tool, the open code movement role in information and documentation infrastructure development, the digital revolution in mass media, and the role of the information technology in the task of dynamizing social networks.

Keywords: Information Science. Research trends. Editorial. Review. Intranets. Free software. Mass media. Public administration. Socio-technological networks. Mexico. Spain.

\section{Introducción}

Este libro presenta un conjunto de trece trabajos que exploran distintos ámbitos de la documentación digital - esto es, la aplicación de la tecnología digital a la documentación o, reciprocamente, de los principios y técnicas documentales a proyectos de información digital- . La documentación digital constituye, junto con la planificación y evaluación de sistemas, el área de las Ciencias de la Documentación que está recibiendo mayor atención en los últimos años, en consonancia con las oportunidades abiertas por los avances de la Informática y las Telecomunicaciones, $\mathrm{y}$, muy en concreto, de la red Internet.

Los artículos son fruto del trabajo de miembros de varios de los grupos de investigación que destacan en nuestro país sobre el tema - el Área de Biblioteconomía y Documentación de la Universidad Autónoma de Barcelona, el Centro de Información y Documentación Científica del Consejo Superior de Investigaciones Científicas, el Grupo de Investigación en Documentación Multimedia de la Universidad Complutense y de la Universidad de Extremadura, el Grupo de Investigación en Recuperación de la Información de Salamanca y el Grupo de Tecnologías de la Información de la Universidad de Murcia-. Además cuenta con las contribuciones del importante grupo de investigación en bibliotecas digitales de la Universidad de las Américas (Puebla, México), y de investigadores de la Universidad Oberta de Catalunya y de Zaintec. Es cierto que, para ser completos tendría que contar con la participación de otros grupos importantes como los de las universidades Carlos III, Extremadura, Granada o Pompeu Fabra, entre otros. Este volumen quiere servir de homenaje a todos ellos, presentes y ausentes, y a los investigadores individuales que trabajan en esta frontera de la investigación sobre Documentación.

Scire. $10: 2$ (jul.-dic. 2004) 9-24. 
Precisando su ámbito temático, los artículos constituyen un panorama representativo de las investigaciones sobre documentación digital tanto desde el punto de vista de sus principales campos de aplicación - las Intranets corporativas, las bibliotecas digitales, los portales científicos y periodísticos, las redes de vigilancia tecnológica, los servicios de información administrativa - como en lo que concierne a algunos de los temas transversales más candentes en la actualidad - el desarrollo de modelos especializados de sistemas de información, el software libre, la transición al XML y a la Web semántica, la búsqueda en lenguaje natural, los metadatos, la visibilidad, la usabilidad, los estudios de usuarios y la minería de datos en la Internet-.

Aunque los temas se solapan, hemos organizado esta presentación en torno a cuatro énfasis: las intranets como instrumento para la gestión del conocimiento, el movimiento del código abierto en el desarrollo de infraestructuras documentales, la revolución digital en la documentación de medios de comunicación, y el papel de la tecnología como elemento dinamizador de proyectos de trabajo en grupoy de animación de redes sociales.

\section{Las intranets al servicio de la gestión del conocimiento}

Uno de los aspectos decisivos de la revolución de Internet ha sido la utilización desde el principio de sus mismas tecnologías de base para facilitar el intercambio de información dentro de las organizaciones. Es lo que se conoce como intranet. Las intranets han aportado a la revolución de la Intranet un énfasis en la integración de servicios y productos en auténticos sistemas de información y documentación - esto es, realmente "sistemáticos" y "sistémicos"-, y la han entroncado con movimientos empresariales como la gestión de la calidad y, de manera muy especial, la gestión del conocimiento, de la que constituyen la infraestructura tecnológica por antonomasia.

José Luis González Ugarte (2004) aborda precisamente en un clarificador y completo trabajo de síntesis las Intranets como instrumento de gestión del conocimiento corporativo; y analiza sus características principales, su tipología, sus ventajas e inconvenientes, los factores de éxito en su desarrollo, sus limitaciones y los retos a los que se enfrenta su implementación eficaz. Ugarte parte de las ideas de Alavi (2001) para precisar cuatro tipos de énfasis en la utilización de la intranet según se oriente respectivamente a la creación, almacenamiento y recuperación, intercambio o uso del conocimiento, siendo éste último la síntesis de los anteriores. A partir de allí, plantea las muchas ventajas que ofrece la intranet para la gestión del conocimiento en las organizaciones, entre las que destaca su capacidad para facilitar un contexto sociotecnológico que hace posible el intercambio y captura del conocimiento tácito; así como la estructuración, almacenamiento, recuperación y visibilidad del conocimiento de la organización en su conjunto.

Scire. $10: 2$ (jul.-dic. 2004) 9-24. 
Las teoría de las tres perspectivas de Stenmark (2002) - información en el sentido de recuperabilidad, conciencia y comunicación - permite observar desde otro punto de vista como se realiza el potencial de la intranet al servicio de la organización. Dentro del cuidadoso estado de la cuestión de los factores de éxito en el diseño de una intranet que realiza Ugarte, enfatizaría de modo muy especial tres condiciones que recoge el autor: la intranet debe ser, dicho en nuestra propia terminología, sistémica - esto es, debe diseñarse para toda la organización, pensando, como señala Ugarte, antes en los usuarios y sus interacciones que en la tecnología-; la cultura organizacional y el capital social deben cultivarse con tanto mimo como la tecnología; y su diseño y mantenimiento deben desarrollarse dentro de los parámetros del paradigma de la gestión de la calidad total, dicho en palabras del autor (ibidem, 2004, p. 37):

Debemos introducir un sistema de calidad en el mantenimiento y desarrollo de las intranets. Un nivel óptimo de calidad y definición de las necesidades funcionales - actualizaciones, supervisión de enlaces, presentación y calidad de contenidos-, y desarrollar guías de la intranet, actualización de los procesos y una evaluación sistemática.

\section{El papel de los estándares y la movimiento del código abierto}

El trabajo de José Ramón Pérez Agüera y Rodrigo Sánchez Jiménez (2004) sirve de introducción al conjunto de estudios que presentamos a continuación. Estos dos jóvenes autores analizan el concepto y características del software libre, sus implicaciones para diferentes tipos de usuarios y su aplicación en Documentación. Tras identificarlo como uno de los temas "calientes" de la actualidad científica y comercial, se aplican a clarificar el cúmulo de imprecisiones que plagan el concepto: El aspecto distintivo del software libre no es la gratuidad, sino el hecho de que su código fuente no está encriptado, al contrario está disponible para ser modificado y adaptado. Por ello, el software libre permite que amplias comunidades de usuarios y programadores lo sometan a escrutinio y experimentación - con toda las posibilidades de intercambio que les ofrecen las comunidades virtuales -, lo que aporta en principio una ventaja evolutiva a este tipo de aplicaciones gracias a la diversidad de su base. Por otra parte, el software libre no da al traste con la libre empresa, pues las organizaciones que realizan actividades comerciales pueden centrarse en la consultoría, adaptación, mantenimiento y formación de sus clientes en vez de en la explotación de la licencia propietaria. Su carácter abierto y sometido al escrutinio comunitario lo convierte en especialmente apto para la educación y la investigación, razón por la que, precisamente, este movimiento se originó y se ha desarrollado especialmente en estos ámbitos. De alguna manera, se le podría considerar la madurez social y legal de la práctica normal de los programadores académicos.

Scire. $10: 2$ (jul.-dic. 2004) 9-24. 
Siguiendo la enumeración Pérez y Sánchez, las aplicaciones concretas en el mundo de la documentación son muy importantes y van desde las herramientas de carácter infraestructural - por ejemplo, sistemas operativos como Linux, gestores de bases de datos como MySQL y PstgreSQL o servidores web como Apache - hasta herramientas especializadas para la gestión de portales y contenidos - PostNuke, Jboss Portal o Mambo - , de bibliotecas virtuales - Phronesis o Greenstone-, de recuperación de información - Isite, "Java Search Engine" de Javaproxy y NGO Search — o la publicación de revistas electrónicas - Public Knowledge-.

Precisamente dentro de los esfuerzos por desarrollar código libre y abierto en el entorno universitario español y en ámbito de nuestra disciplina, merece la pena destacar el trabajo desarrollado por el Grupo de investigación en Recuperación de la Información Automatizada (REINA) de la Universidad de Salamanca. Sus miembros - Carlos García Figuerola, José Luis Alonso Berrocal, Emilio Rodríguez y Ángel F. Zazo Rodríguez (2004a y 2004b) - publican dos trabajos complementarios en los que presentan respectivamente el desarrollo y evaluación del motor de búsquedas que han desarrollado para realizar experimentos de recuperación de información dentro del marco de la Text Retrieval Conference (TREC) - el principal foro mundial en recuperación de información textual-. El motor - llamado Karpanta - está disponible en Internet para acceder a una base de datos real sobre Ciencias de la Documentación, y ha sido utilizado también como motor de Internet en otros proyectos de carácter específico como la evaluación de las páginas de investigación de las universidades españolas (Pinto et al., 2004, 2005). El motor está especialmente dirigido a la recuperación en lenguaje natural basada en el modelo vectorial, implementa más de trescientos algoritmos frecuentemente usados en documentación, e incorpora también el control de palabras vacías y la lematización en el proceso de indización. En consonancia con su carácter de herramienta de investigación y docencia, es un paquete de código libre y abierto con licencia General Public License (GPL) que puede ser utilizado, modificado y adaptado libremente por cualquier investigador. Esta programado mediante sentencias SQL sobre SQlite - un servidor y entorno de programación SQL también libre y portable-, por lo que resulta fácilmente interpretable y asequible. Además, se separan los procesos de indización y recuperación en módulos diferenciados para facilitar la realización de cambios controlados. Se trata de una excelente herramienta para la investigación que apenas ha empezado a dar sus primeros frutos.

En el capítulo siguiente Ángel F. Zazo Rodríguez, Carlos García Figuerola, José Luis Alonso Berrocal y Emilio Rodríguez (2004b) presentan el análisis de los archivos de registro de la implementación en línea del sistema de recuperación Karpanta que el equipo ha realizado con una biblioteca de documentos en texto

Scire. $10: 2$ (jul.-dic. 2004) 9-24. 
completo de nuestra especialidad. La investigación ha sido desarrollada como un modelo del análisis de la interacción entre usuarios y sistemas en el contexto de las bibliotecas digitales, y ciertamente ofrece datos muy interesantes sobre el comportamiento de las personas que realizan las búsquedas. Entre los aspectos de carácter general, se estudian la procedencia - a partir del estudio de las direcciones IP y sus dominios y subdominios - de las consultas, su distribución temporal y su número por usuarios, tanto en total como en le marco de cada sesión. De gran interés son los análisis de las características de las consultas realizadas por los usuarios, que validan resultados obtenidos en otros estudios. El número medio de términos por consulta es de 2,66; un valor muy parecido al que reflejan otros estudios sobre bibliotecas digitales y motores de búsqueda en Internet. Los usuarios incumplen en casi un diez por ciento de los casos las instrucciones de uso, intentando utilizar operadores que no están disponibles en el sistema. Es significativo que entre los operadores erróneamente utilizados no se encuentre el de exclusión y que casi el $20 \%$ de las consultas repetidas sean exactamente iguales que la anterior. Todo esto indica la existencia de un conjunto de usuarios muy notable con escasas habilidades de recuperación. Por el contrario, algo más de la mitad de los usuarios modifica las opciones por defecto de visualización y limitación de las búsquedas; y el 40,43\% de las consultas repetidas son reformulaciones de la anterior, pues contienen al menos un término en común (ibidem, p. 75):

Visto que la reformulación de consultas es una practica habitual, y que el número medio de términos por consulta es de 2,66, parece claro que los usuarios refinan sus necesidades informativas con cambios muy pequeños: añadiendo algún término, modificando algún otro, quitando otro, etc.

Por último, los autores nos presentan algunas de sus futuras líneas de trabajo: el análisis del comportamiento de visualización y el de retroalimentación de consultas mediante la implementación en Karpanta de procedimientos de visualización más avanzados; la inclusión de la función de búsqueda de páginas semejantes; y la implementación de la realimentación por relevancia a partir de la selección por el usuario de referencias óptimas.

Otro interesantísimo proyecto de software abierto, en este caso de bibliotecas digitales y en conformidad con los estándares preconizados por la Iniciativa de Archivos Abiertos (Open Archives Innitiative), es el presentado por Patricia García Jiménez, José Alfredo Sánchez y Alberto García García (2004), bibliotecarios y profesores de la Universidad de las Américas de Puebla (UDLA, México). En realidad, el proyecto "Red Nacional de Acervos Antiguos Digitalizados", una extensión del programa de Bibliotecas Digitales Universitarias para Todos (UDLA), que desarrolla la UDLA, va más allá del desarrollo de software y pretende también la publicación de contenidos. El proyecto consiste en la creación de una biblioteca digital federada de libros antiguos pertenecientes a colecciones

Scire. $10: 2$ (jul.-dic. 2004) 9-24. 
mexicanas con el objetivo de favorecer su preservación, y su difusión y consulta a través de Internet. Incluye el diseño e implementación de componentes de aplicaciones de consulta, visualización y navegación de colecciones especiales digitalizadas, así como de recuperación de información en texto completo. Efectivamente, las bibliotecas digitales constituyen el procedimiento de elección para difundir las colecciones antiguas, porque facilitan el acceso con todas las ventajas de la Internet sin comprometer la conservación, pues no es necesario acceder al ejemplar original salvo en ocasiones especiales (ibidem, p. 77-78):

La preservación de acervos bibliográficos históricos ha cobrado importancia mundial a partir de su valoración como elementos clave en el desarrollo de la humanidad. La belleza de los libros antiguos, aunada a la riqueza de su contenido, los convierte en obras de arte dignas de admirar, conservar y estudiar. En México, diversas bibliotecas que cuentan con libros y archivos antiguos se han sumado a la gran tarea de rescatar, catalogar y difundir tan preciados materiales. Desafortunadamente, el gran valor y en muchas ocasiones el deterioro de estos libros hacen necesario restringir el acceso a los acervos rescatados y minimizar su manipulación física. Con ello, su uso y divulgación se logran solamente de manera muy limitada. Actualmente y gracias al desarrollo de la tecnología, los responsables de los repositorios que custodian colecciones históricas pueden evitar el daño material sobre documentos antiguos mediante proyectos de digitalización, que a la vez proporcionan acceso y difusión universal. A través de la implementación de las llamadas bibliotecas digitales, se crean herramientas que facilitan la consulta de documentos y su aprovechamiento para la investigación y posterior creación de conocimiento.

En el ámbito del aprovechamiento de los estándares abiertos, Blanca Gil Urdiciain, José Ramón Pérez Agüera y Rodrigo Sánchez Jiménez (2004) analizan el módulo de metadatos de Synchronized Multimedia Integration Language (SMIL) - una recomendación propuesto por el World Wide Web Consortium - y ejemplifican el uso. Como afirman los autores (ibidem, p. 87-88):

El camino hacia la Web Semántica pasa por la identificación unívoca, clara y concisa de cada una de las unidades informativas que la componen. En el caso de la documentación multimedia esto es especialmente delicado, ya que cada documento puede estar compuesto a su vez por otros documentos que - si seguimos la afirmación anterior - deben a su vez poder ser identificados tanto por separado como en conjunto de cara a su recuperación posterior.

SMIL está basado en el Extensible Markup Language (XML). Es, por lo tanto, una propuesta abierta e interoperable para la integración de elementos multimedia, que funciona de la forma modular y etiquetada a la que nos ha acostumbrado el Hypertext Markup Language (HTML) —el desarrollo más popular de los lenguajes de etiquetado de propósito general cuyo marco codifica la norma Standard Generalized Markup Language (SGML) - . El módulo permite la adopción de un esquema de metadatos concretos, como pueda ser la Dublin Core

Scire. $10: 2$ (jul.-dic. 2004) 9-24. 
Metadata Initiative (DC) (2005) u otros más específicos como la Video Metadata Representation. Lo más importante es que permite describir los elementos componentes de cualquier documento compuesto y multicanal (Pinto Molina, García Marco y Agustín Lacruz, 2002), asegurando así un aumento de la especificidad y exhaustividad de la recuperación y la reutilización de los contenidos en nuevos productos comunicativos.

\section{La revolución digital en los medios de comunicación}

El subconjunto de estudios temáticamente homogéneos más amplio corresponde al ámbito de los medios de comunicación. Los artículos abordan de manera muy especial la evaluación de los portales de los medios en aspectos tales como la visibilidad, la usabilidad o la oferta de servicios.

En el campo de la visibilidad destaca el proyecto que está llevando a cabo el Área de Documentación de la Universidad Autónoma de Barcelona sobre la autodescripción que la prensa local y regional española realiza de sus ediciones en Internet. Los webs periodísticos - especialmente los de la prensa - han evolucionado hasta configurar importantes portales de información, referencia y servicios en sus ámbitos de difusión. Por ello, resulta fundamental asegurar la recuperación de esa información en el espacio global que configura la Red. En particular, los miembros del equipo - Àngels Jiménez, Eulàlia Fuentes y Alfons González (2004) - presentan en este trabajo los resultados de una investigación sobre el uso que una muestra representativa de veintitrés diarios locales y regionales españoles realizan de las metaetiquetas, con especial atención a las dedicadas a la descripción del contenido, esto es, 'description' y 'keywords' .

Los autores se hacen eco de la desconfianza de los grandes motores de búsqueda hacia las metaetiquetas - debido al abuso que se ha realizado de la autodescripción para posicionar las páginas en los buscadores, y que llegó hasta el extremo de asignar a las páginas términos muy buscados que no tenían nada que ver con su contenido real-. Sin embargo, constatan el interés de los diarios en hacerse presentes por medio de esta tecnología, ya que la mayoría de ellos sigue actualizando las metaetiquetas. Detectan, eso sí, la ausencia de un esquema de metadatos estándar que dé homogeneidad al tipo y al contenido de las metatags de cada diario analizado. Por el contrario, se aprecia el avance de una cierta normalización lingüística a través de la utilización del inglés.

Por lo demás, llegan a interesantes conclusiones sobre el procedimiento de indización seguido por los diarios. La indización se centra en la página inicial de la sede web del medio de comunicación, y fluctúa entre la descripción de sus características generales - estructura de contenidos y oferta de servicios de valor añadido- y la inclusión de términos referentes a las noticias más impactantes del

Scire. $10: 2$ (jul.-dic. 2004) 9-24. 
momento con la intención - aquí también - de posicionar al diario en los buscadores. Más allá de su interés inmediato para el estudio visibilidad de la prensa en Internet, el cuidadoso análisis que realizan Jiménez, Fuentes y González de las características de la descripción de contenido efectuada por los diarios tiene un gran valor metodológico para el estudio de la indización en entornos especializados.

A continuación se presentan sendos trabajos de dos miembros de otro de los grupos de investigación de referencia dentro del campo de la investigación documental digital en nuestro país: el Grupo de Tecnologías de la Información de la Universidad de Murcia. En el primero de ellos, Rosana López Carreño (2004) aborda el estudio de las sedes web de los medios de comunicación españoles de radio y televisión con el objetivo de analizar su transformación en portales de servicios y de proponer un modelo de referencia que facilite su evaluación y planificación. Se trata de una extensión a estos medios del modelo propuesto por la autora para los portales periodísticos de prensa (2003). Una primera conclusión que ofrece es el grado de inferior desarrollo que los portales de radio y televisión han alcanzado respecto a los de prensa, aunque se constata su rápido avance siguiendo, en buena parte, el modelo marcado por éstos (ibidem, p. 121):

$\mathrm{Al}$ igual que sucede en los portales periodísticos de prensa, predominan los productos informativos frente a los documentales [...], destacan por su frecuencia de uso las categorías de servicios de valor añadido de acceso a la información y de participación. De modo semejante sucede en los portales de origen prensa, donde los SVA de acceso a la información se alzan como los servicios más ofertados, aunque los interactivos se colocan por delante de los de participación.

Constituye una cierta aporía - aunque comprensible y reveladora del estado actual real de la sociedad de la información multimedia-, que los portales de radio y televisión abunden en información textual, pero adolezcan de escasa información en vídeo y audio. La difusión por Internet de estos contenidos y la integración de la Internet con otros canales utilizados para su difusión — notablemente los operadores de cable - constituye precisamente una de las fronteras de las industrias de los contenidos. Por otra parte, la rápida proliferación de herramientas de producción muy avanzadas destinadas al usuario normal - como el software iLife - y la bajada de precio del hardware terminará por provocar en el mundo multimedia una explosión semejante a la que produjo en el ámbito de la información textual la generalización del proceso de textos.

El otro miembro del Grupo de Tecnologías de la Información de la Universidad de Murcia que participa en el número monográfico, Juan Carlos García Gómez (2004), aborda el estudio de la usabilidad de páginas web. García Gómez traza con habilidad las líneas maestras de la discusión sobre la usabilidad. Frente a los diseños efectistas que buscan atrapar la atención del usuario dentro

Scire. $10: 2$ (jul.-dic. 2004) 9-24. 
del paradigma del escaparate, los teóricos de la usabilidad plantean que, una vez que el usuario ha llegado a la página - lo cual presupone que su atención ya ha sido de alguna manera captada con anterioridad en un buscador, catálogo o portal-, lo importante es que pueda acceder fácilmente a la información y los servicios ofrecidos y que pueda sacar provecho de ellos. Más allá de la eficaz y obvia definición de Nielsen (2003) - "Usabilidad es un atributo de calidad que mide lo fáciles de usar que son las interfaces web" -, García Gómez trae a colación también la - quizá más operativa - de Redish (2000), para el que la usabilidad consiste en diseñar sitios web que permitan a los usuarios "encontrar lo que necesitan, entender lo que encuentran y actuar apropiadamente... dentro del tiempo y esfuerzo que ellos consideran adecuado para esa tarea", lo que se consigue respetando los principios de la ergonomía perceptiva y cognitiva, y las normas y usos comunes de la web y de la publicación en general.

Centrándonos en su aportación al estudio de la Documentación de medios, García Gómez estudia las páginas de inicio de los principales diarios españoles de información general -El País, El Mundo, ABC, La Razón-, los quince diarios en línea de información general más visitados en el 2003 según la Oficina para la Justificación de la Difusión y dos diarios solo en Internet - Estrella Digital y Diario Directo - . Para ello utiliza una parrilla de análisis basada en una adaptación de las propuestas de usabilidad de Nielsen, que completa con algunos ítems - presencia de un título significativo en la página, la presencia o no de pop-ups, la inclusión de un eslogan adecuado, el etiquetado de imágenes y el uso o no menús desplegables - y un mecanismo de ponderación que le permite, en definitiva, ofrecer una puntación comparativa. Los resultados obtenidos muestran niveles aceptables, puntos débiles y fuertes y algunas tendencias consolidadas, que el autor resume así (ibidem, p. 135):

En primer lugar, todos los webs estudiados tienen un tamaño excesivo - en torno a $250 \mathrm{~kb}-$, lo que implica un tiempo de descarga excesivamente lento para terminales equipados con conexiones a la red lentas. En segundo lugar, no se suelen emplear los estándares web para los links, aunque normalmente éstos se distinguen perfectamente gracias al color y tipografía utilizados. En tercer lugar, las webs de diarios en línea pertenecientes a grupos empresariales medio-bajos tienden a no ofrecer buscador. En cuarto lugar, el ancho de página siempre está optimizado a 800x600 (ancho fijo), en formato de cuatro columnas. La primera de ellas se reserva a la navegación principal, las dos centrales contienen las noticias y la cuarta alberga la navegación secundaria y el acceso a servicios de valor añadido. En quinto lugar, los resultados muy bajos tienden a deberse en su mayoría a deficiencias en los sistemas de ayuda a la navegación, rotulación e información de contacto.

También muestran una aparente paradoja - los diarios más usables no son los de mayor difusión, sino los vinculados a grupos empresariales de tamaño intermedio-, que García Gómez explica así (ibidem, p. 134):

Scire. $10: 2$ (jul.-dic. 2004) 9-24. 
Las páginas de inicio de los diarios en línea tienden a ser tanto más usables cuanto más sencillo sea su diseño. El minimalismo de éste tiende a orientar al usuario a lo esencial (los contenidos), evitando distraerle con elementos secundarios o provocarle retrasos en el acceso a la información. Por el contrario, el exceso de sofisticación en el diseño de una web en busca de la originalidad tiende a la construcción de sitios web en los que no se respetan los estándares y los usos más frecuentes en Internet, lo que termina por hacer ese sitio menos usable. [...] Esto, por tanto, explicaría que los mejores resultados obtenidos correspondan a las páginas de inicio de webs de grupos empresariales con presupuesto medio-bajo, en los que se optó por un diseño sin exceso de sofisticación.

Efectivamente, la disponibilidad presupuestaria alta puede jugar en contra de la usabilidad, pues favorece - o al menos permite - la complejidad y el uso de tecnologías no estándar. En una línea semejante, se aprecia también como el éxito en visitas suele conllevar la tentación de aumentar la publicidad, lo que satura la página y la hace igualmente menos usable. La sencillez de diseño - que muchos, entre ellos nuestro autor, etiquetan como minimalismo - se muestra también aquí como una técnica que facilita la adopción y uso de la tecnología, cosa que sobradamente han demostrado fabricantes como Apple Computer.

Finalmente, Jorge Caldera Serrano, María Victoria Nuño Moral y Felipe Zapico Alonso (2004) presentan, describen y analizan la información audiovisual proporcionada por las agencias de noticias internacionales -Eurovisión, Associated Press y Reuters - desde el punto de vista documental, mostrando las diferentes peculiaridades físicas y formales que la definen como fuente de información para las empresas televisivas, atendiendo especialmente a las características de los titulares y la calidad de las imágenes. Los autores nos acercan con maestría al trabajo de estas organizaciones y explican su función. Las agencias de noticias realizan un papel fundamental, pues a través de sus redes consolidadas y diversificadas permiten el acceso a la noticia a un precio aceptable, que luego debe integrarse en el activo informacional de la organización mediante "políticas claras de adquisición, selección, tratamiento y conservación". Además, aseguran un flujo de noticias puntual y estandarizado. El conjunto de metadatos que utilizan estas organizaciones es, por lo demás bastante lógico:

La información aportada por las agencias, aunque escasa para la descripción del material audiovisual, sí que está prácticamente estandarizada, y muestra los aspectos geográficos - lugar de la acción-, temáticos - palabras en texto libre que identifican su contenido-, físicos - duración - y legales - restricciones de acceso a la información-.

\section{El papel dinamizador de las administraciones públicas}

A continuación se presentan tres excelentes ejemplos del papel dinamizador que cabe a las administraciones publicas en diversas áreas de la vida económica

Scire. $10: 2$ (jul.-dic. 2004) 9-24. 
y social apoyándose en las tecnologías de la información y la comunicación. Se trata de tres proyectos realizados respectivamente en el campo de la información científica, la información juvenil y la información estratégica en el Centro de Información y Documentación Científica, el Gobierno de Aragón y la Diputación Foral de Vizcaya.

En el primer trabajo, Elena Fernández (2004) presenta el portal Tecnociencia como ejemplo de una iniciativa llevada a cabo por la administración central para potenciar la difusión y la transferencia de los resultados del trabajo científico y tecnológico llevado a cabo en España y en menor medida en el ámbito europeo a todos los sectores de la sociedad. El artículo presenta las características de este portal: su estructura básica, los canales temáticos, los diferentes recursos de información referencial, contenidos monográficos — denominados "singulares" - y los servicios que se ofrecen a los usuarios. Se trata de un trabajo modélico desarrollado por un amplio equipo interdisciplinar de especialistas, fruto de la colaboración entre el Centro de Información y Documentación Científica (CINDOC) - perteneciente al Consejo Superior de Investigaciones Científicas (CSIC) - y el Ministerio de Ciencia y Tecnología. A parte de proporcionar una información de carácter enciclopédico y recursos de todo tipo, el portal cuida mucho los aspectos de interactividad real, pues su objetivo es "abrir una "ventana de la ciencia" donde los usuarios puedan formular sus dudas y preguntas sobre cualquier tema de su interés relacionado con la Ciencia y la Técnica, así como [...] enviar sus colaboraciones y trabajos para su publicación en el web. En definitiva, se busca expresamente la colaboración de los usuarios." (ibidem, p. 124).

El segundo proyecto - denominado "Infojoven.com" - ha sido desarrollado por José Juste Jordán, Lorena Monge Vázquez y Daniel Pons Vertían (2004) en el Instituto Aragonés de la Juventud, y aborda la cooperación, intercambio y difusión selectiva de información juvenil. Su objetivo es el desarrollo una red de trabajo horizontal, libre y voluntaria que permita compartir contenidos y crear herramientas de explotación del conocimiento, siguiendo los principios de la "carta Eryica" y conectando con la filosofía del software libre y diversos movimientos alternativos que movilizan a parte de los jóvenes. Está orientado a los profesionales de la información juvenil que suministran información específica a los jóvenes - los usuarios finales -, con el objetivo de optimizar recursos compartiendo información validada y verificada. En consonancia con su filosofía abierta, cooperativa y orientada al trabajo en red, el sistema de información se basa en una arquitectura cliente-servidor abierta, y se ha cuidado especialmente el desarrollo de un módulo para distribuir la información mediante una central de comunicaciones multicanal.

En el tercero, Sonia Saénz de Lacuesta Sáez de Ocáriz y Maitane Bilbao Arruza (2004) presentan la metodología y herramientas de desarrollo de un servi-

Scire. $10: 2$ (jul.-dic. 2004) 9-24. 
cio de vigilancia tecnológica e inteligencia competitiva para pequeñas y medianas empresas (PYME) que se apoya en un portal de Internet y en una amplia red de colaboración entre usuarios, agentes y expertos de vigilancia tecnológica. El servicio - denominado Zaintek - es una iniciativa del Departamento de Innovación y Promoción Económica de la Diputación Foral de Vizcaya. El proyecto está documentado en un manual muy interesante, e incluye un panel de indicadores de resultados. Desde el punto de vista tecnológico, se apoya en dos aplicaciones de gestión soportadas por Internet (ibidem, 168):

[...] a) la aplicación web para el uso de las empresas miembro de Zaintek, en la que se pueden realizar consultas, solicitar alertas, sistemas de información empresarial y se puede acceder a otro tipo de información como son las noticias, debates, biblioteca, etc.; y b) la aplicación de gestión externa extranet, en la que existen dos figuras fundamentales que son el coordinador - encargado de realizar algunas acciones como la aceptación de las ofertas de los expertos, la distribución de las solicitudes de las empresas a los expertos, etc. - y el experto, que es el que normalmente realiza ofertas y genera las respuestas a las empresas.

\section{Conclusiones}

Para los profesionales de la información la cultura digital y sus herramientas no son un fin en sí mismas, sino, respectivamente, el entorno de trabajo y de referencia en el momento actual, y un medio para conseguir los fines que tienen encomendados; esto es, asegurar el acceso y la preservación de los registros informativos en condiciones idóneas para las organizaciones, comunidades y sociedades para las que trabajan. Para ello desarrollan iniciativas tanto de carácter restringido - Intranets y extranets - , como proyectos de acceso público en la Internet - servicios de información y bibliotecas digitales públicas-.

Es lógico que, desde el punto de vista de sus fines a largo plazo, los profesionales de la información muestren una preferencia natural por aplicaciones que se conformen a los estándares generalmente aceptados, que tengan posibilidad de durar en el medio y largo plazo, que proporcionen seguridad y autenticidad documental, y que tengan un carácter abierto e interoperable. No son ingenuos y comprenden que, lógicamente, las aplicaciones líderes sean por lo general cerradas para facilitar el retorno de la inversión y asegurar su competitividad. Esto es una consecuencia del libre mercado y, en el fondo, un mecanismo aceptado por la sociedad para favorecer la innovación y la responsabilidad en las iniciativas económicas. Lo que va comprendiéndose cada vez con más claridad es que no debe permitirse que los formatos de almacenamiento e intercambio tengan en sí mismos carácter propietario, antes al contrario: deben ser decididamente abiertos e interoperables para no poner en peligro los fines del propio trabajo informativodocumental. 
Por otra parte, conforme la tecnología avanza y se generaliza es lógico que se produzca una nivelación en la tecnología y que el código de las aplicaciones se generalice a través de distintos procedimientos totalmente legales, como el fin de las limitaciones del derecho de copia o la codificación de las funcionalidades mediante procedimientos innovadores libremente disponibles que no violen derechos vigentes. Es lógico que esto suceda y además parece justo, en la medida en que muchas innovaciones que utilizan las empresas se han producido también en el sector de investigación público o en relación con él. Este fenómeno de nivelación es lo que está ocurriendo con la aparición de excelentes aplicaciones de carácter gratuito que ofrecen para entornos pequeños y medianos las funcionalidades de programas comerciales muy caros, en campos tan diversos como los que van desde los gestores de bases de datos y servidores web hasta las infraestructuras para gestión de contenidos y bibliotecas digitales. Su gratuidad es, lógicamente, relativa, porque permanecen los costos de operación, normalmente muy altos al implicar a personal muy especializado.

Este complejo motor de intercambios entre la iniciativa privada y la investigación pública está ayudando a atender distintos sectores del mercado - algunos de los cuales simplemente no podrían acceder a estas infraestructuras - y, en definitiva, está colaborando en la difusión de las tecnologías de la información, la comunicación y la documentación en el conjunto de la sociedad mundial, lo cual, en distintas maneras, redunda en beneficio de todos: amplía el mercado y contribuye al desarrollo. Como moderar las consecuencias indeseables de este proceso - en todas las direcciones - , es otro asunto que merece la máxima atención.

\section{Referencias}

Alavi, M.; Leidner, D. E. (2001). Knowledge Management and Knowledge Management Systems: Conceptual Foundations and Research Issues. // MIS Quarterly. 25:1 (2001) 107-136.

Caldera Serrano, Jorge; Nuño Moral, María Victoria; Zapico Alonso, Felipe (2004). Las agencias internacionales de noticias como fuente de información audiovisual. // Scire: representación y organización del conocimiento. 10:2 (jul.-dic. 2004) 137-146.

Fernández Sánchez, Elena (2004).El portal Tecnociencia: un sistema de Información Científica en Ciencia y Tecnología. // Scire: representación y organización del conocimiento. 10:2 (jul.-dic. 2004) 147-154.

García Figuerola, Carlos; Alonso Berrocal, José Luis; Zazo Rodríguez, Ángel Francisco; Vázquez de Aldana, Emilio Rodríguez (2004).Herramientas para la investigación en Recuperación de Información: Karpanta, un motor de búsqueda experimental. // Scire: representación y organización del conocimiento. 10:2 (jul.-dic. 2004) 51-62.

García Jiménez, Patricia; Sánchez; José Alfredo; García García, Alberto (2004). Acervos antiguos digitales: hacia una red nacional mexicana. // Scire: representación y organización del conocimiento. 10:2 (jul.-dic. 2004) 123-136.

Scire. $10: 2$ (jul.-dic. 2004) 9-24. 
Gil Urdiciain, Blanca; Pérez Agüera, José Ramón; Sánchez Jiménez, Rodrigo (2004). Gestión de documentación multimedia con Synchronized Multimedia Integration Language (SMIL). // Scire: representación y organización del conocimiento. 10:2 (jul.-dic. 2004) 87-97.

González Ugarte, José Luis (2004). Gestión del conocimiento en intranets corporativas: desarrollo de un modelo de diseño. // Scire: representación y organización del conocimiento. 10:2 (jul.-dic. 2004) 25-39.

Jiménez, Àngels; Fuentes, Eulàlia; González, Alfons (2004).Aproximación al estudio de la visibilidad de la prensa local y regional en Internet: el análisis de las metatags. // Scire: representación y organización del conocimiento. 10:2 (jul.-dic. 2004) 99-113.

Juan Carlos García Gómez, (2004). Usabilidad de las páginas de inicio de los diarios digitales españoles. // Scire: representación y organización del conocimiento. 10:2 (jul.-dic. 2004) 123-136.

Juste Jordán, José; Monge Vázquez, Lorena; Pons Betrián, Daniel (2004). Sistema de Localización de Recursos de Información: (SLRI): "Infojoven.com”. // Scire: representación y organización del conocimiento. 10:2 (jul.-dic. 2004) 155-166.

López Carreño, Rosana (2004). Grado de desarrollo de los portales periodísticos españoles de radio y televisión. // Scire: representación y organización del conocimiento. 10:2 (jul.-dic. 2004) 115-122.

Nielsen, Jakob; Tahir, M. (2002). Usabilidad de páginas de inicio: Análisis de 50 sitios web. Madrid: Prentice Hall, 2002.

Pérez Agüera, José Ramón; Sánchez Jiménez, Rodrigo (2004). Documentación y software libre . // Scire: representación y organización del conocimiento. 10:2 (jul.-dic. 2004) 41-49.

Pinto Molina, Maria; Alonso Berrocal, José Luis; Cordón García, José Antonio; Fernández Marcial, Viviana; García Figuerola, Carlos; García Marco, Francisco Javier; Camarero, Carmen Gómez; Zazo, Ángel Francisco; Doucet, Anne-Vinciane (2004). Análisis cualitativo de la visibilidad de la investigación de las universidades españolas a través de sus páginas web. // Revista Española de Documentación Científica. 27: 3 (jul.-sept 2004) 245-370.

Pinto Molina, Maria; Alonso Berrocal, José Luis; Cordón García, José Antonio; Fernández Marcial, Viviana; García Figuerola, Carlos; García Marco, Francisco Javier; Camarero, Carmen Gómez; Zazo, Ángel Francisco (2005). Quality Assessment of Spanish Universities web sites focused on the European Research Area. // Scientometrics. 65:1 (October, 2005).

Pinto Molina, María ; García Marco, Francisco Javier ; Agustín Lacruz, María del Carmen (2001). Indización y resumen de documentos digitales y multimedia : técnicas y procedimientos. Gijón: Trea, 2001.

Saénz de Lacuesta Sáez de Ocáriz, Sonia; Bilbao Arruza, Maitane (2004). Integración de agentes regionales de innovación y prestación de servicios avanzados de vigilancia tecnológica e inteligencia competitiva para PYMEs: el caso Zaintek. // Scire: representación y organización del conocimiento. 10:2 (jul.-dic. 2004) 167-171.

Redish, Janice; Dumas, J. (1999). A Practical Guide to Usability Testing. Intellect, 1999. 


\section{Francisco Javier García Marco}

Stenmark, D. (2002a ). Designing the new intranet. (Tesis doctoral). Goteborg: Viktoria Institute, 2002.

Zazo Rodríguez, Ángel Francisco; García Figuerola, Carlos; Alonso Berrocal, José Luis; Vázquez de Aldana, Emilio Rodríguez (2004). El sistema de recuperación Karpanta: estudio de usuarios a través del archivo de registro. // Scire: representación y organización del conocimiento. 10:2 (jul.-dic. 2004) 63-76. 